\title{
Mutation detection of KRAS by high-resolution melting analysis in Chinese with gastric cancer
}

\author{
ZHI-MIN LIU ${ }^{1}$, LI-NA LIU ${ }^{2}$, MEI LI ${ }^{1}$, QIU-PING ZHANG ${ }^{3}$, SHI-HUA CHENG ${ }^{3}$ and SHEN LU ${ }^{1}$ \\ ${ }^{1}$ Laboratory Center, The Second Affiliated Hospital of Dalian Medical University, Dalian 116027; \\ Departments of ${ }^{2}$ Gastroenterology and ${ }^{3}$ Pathology, The First Affiliated Hospital of \\ Dalian Medical University, Dalian 116011, Liaoning Province, P.R. China
}

Received April 30, 2009; Accepted June 15, 2009

DOI: $10.3892 /$ or_00000465

\begin{abstract}
KRAS proteins play an important role in regulating cell functions. A series of studies has revealed that mutations of KRAS are involved in gastric carcinogenesis. However, mutation status of KRAS remains unclear in gastric cancer from Chinese Mainland. It has been proved that KRAS mutation associates with resistance to epidermal growth factor receptor (EGFR) inhibitors. In this study, KRAS mutations were detected in 52 gastric adenocarcinomas from Northern China. High-resolution melting analysis (HRMA) was used and positive samples were confirmed by direct sequencing. Of the 52 cancers, KRAS mutations were found in $5(9.6 \%)$. All cancers with KRAS mutation were from male patients. Frequencies of KRAS mutation were $14.3 \%$ $(3 / 21)$ and $6.5 \%(2 / 31)$ in differentiated and undifferentiated cancers; $25 \%(1 / 4)$ and $8.3 \%$ (4/48) in early and advanced wall penetration cancers; and were $13.3 \%(2 / 15)$ and $8.1 \%$ (3/37) in without and with lymph node metastasis cancers, respectively. There was no significant correlation between KRAS mutation and clinicopathological features. There were 3 mutation types in the 5 mutations, including 2 G12D, 1 G12V and 2 G13D mutations. All codon 12 mutations were found in patients with lymph node metastasis and at advanced stage, whereas all codon 13 mutations were found in patients without lymph node metastasis and at early stage. These results support KRAS mutation may only be involved in carcinogenesis of partial gastric cancers and the different mutation types of KRAS may take part in development of gastric cancer at different stages. The resistance of partial gastric cancer patients to EGFR inhibitors may be induced by KRAS mutation.
\end{abstract}

Correspondence to: Professor Shen Lu, Laboratory Center, The Second Affiliated Hospital of Dalian Medical University, Dalian 116027, P.R. China

E-mail: lushen1956@yahoo.com.cn

Key words: KRAS gene, somatic mutation, gastric cancer, Chinese Mainland

\section{Introduction}

Gastric cancer is the fourth leading cancer in terms of incidence and the second most common cause of cancerrelated death worldwide (1). Chemotherapy is still the main therapeutic approach for gastric cancer patients being at advanced stage in China. Incomplete knowledge on gastric carcinogenesis has slowed down the development of chemical drugs.

In gastric carcinogenesis, abnormal activation of EGFRRAS-RAF-MAP-kinase pathway has been noted (2). In this pathway, activating mutations of EGFR gene can lead to ligand-independent activation of the receptor, resulting in overactivation of downstream effectors. Based on these findings, EGFR inhibitors were developed and used for treatment of some cancers especially non-small cell lung cancer (NSCLC) (3). KRAS, a member of RAS family, plays an important role as a 'molecular switch' in the EGFR-RASRAF-MAP-kinase pathway. Mutations of KRAS can alter the gene conformation and result in constitutive activation of KRAS protein. Generally, KRAS mutations are mutually exclusive to EGFR mutations. Therefore, KRAS mutations can over-activate the EGFR-RAS-RAF-MAP-kinase pathway independent of EGFR mutations. Accordingly, tumors with KRAS mutation will appear resistant to EGFR inhibitors. In clinical usage of EGFR inhibitors for NSCLC patients, KRAS mutation has been confirmed as a negative predictor. It is controversial whether EGFR inhibitors are applicable for gastric cancer, in which the mutation status of KRAS in gastric cancer is a determinant besides mutation status of EGFR.

Since their discovery two decades ago, KRAS mutations have been described in many types of human cancers with significantly different mutation frequency, including pancreas, colon, lung, breast and stomach cancer (4-6). High KRAS mutation frequency was found in many cancers such as pancreas, colon and lung cancer, whereas, gastric cancer has relatively low KRAS mutation frequency. In gastric cancer, the frequency of KRAS mutation varied with human populations $(7,8)$. Previous data of KRAS mutation frequency in gastric cancer from China are few and controversial. According to previous studies, KRAS mutations 
commonly arise at codon 12 and 13 of exon 2 . The spectra of KRAS mutation types of the mutation hotspots were not the same in different kinds of tumors.

High-resolution melting analysis (HRMA) is a recently developed mutation detection technique based on polymerase chain reaction (PCR). It can discriminate mutated from wildtype samples according to the difference between them in the melting curves. The melting curves were generated when PCR amplicons bind with DNA intercalating dyes exposed to increasing temperature. Compared with previous mutation detection methods, HRMA can screen any type of base variation within the whole amplified sequence without postPCR handling. Otherwise, HRMA has the advantages of faster speed, less cost, higher sensitivity. HRMA has been used for detecting somatic and germline mutations in several genes e.g. in EGFR, BRAF and TP53 genes (9-11).

In this study, to clarify the role of KRAS mutation in gastric carcinogenesis and to estimate the applicability of EGFR inhibitors in gastric cancer patients, we detected the mutation status of KRAS gene exon 2 in gastric cancer from Northern China by HRMA.

\section{Materials and methods}

Patients and tissue specimens. A total of 52 fresh resected gastric adenocarcinomas were collected from the hospitals in Dalian region of Northern China. The patients consisted of 36 men and 16 women with a median age of 65 years. None of the patients received radio- or chemotherapy before surgery. Approval of The Ethics Committee of the Dalian Medical University and informed consent of patients were obtained during the procedures of collecting specimens. Two certified pathologists reviewed available $H \& E$-stained slides and diagnosed histological type, differentiation grade of all specimens independently. The status of gastric wall penetration and lymph node metastasis were recorded according to standard criteria and taken as basis for tumor staging. Clinicopathological features of all patients are summarized in Table I. These specimens were diagnosed as well differentiated $(n=3)$, moderate differentiated $(n=18)$, poorly differentiated $(n=26)$ and signet-ring cell cancer $(n=5)$. Among all the 52 specimens, there were 4 with early wall penetration and 48 with advanced wall penetration. Lymph node metastasis was found in 37 gastric cancer patients.

Genomic DNA extraction. Frozen-sections of $5 \mu \mathrm{m}$ thickness and stained with H\&E were made to locate tumor-rich regions by microscope. Tumor-rich regions were isolated for DNA extraction. Genomic DNA was extracted by TIANamp Genomic DNA kit (TIANGEN Biotech, Beijing, China) and kept at $4^{\circ} \mathrm{C}$ before use.

High-resolution melting analysis. A 167 bp amplicon was produced by primers of KRAS exon 2 . The sequences of the primers were 5'-AGGCCTGCTGAAAATGACT-3' (forward) and 5'-AATGGTCCTGCACCAGTAA-3' (reverse). PCR for HRM analysis was performed on the Eppendorf Mastercycler gradient (Eppendorf AG, Hamburg, Germany). A final volume of $10 \mu 1$ mixture contained: 10X PCR buffer (Takara,
Dalian), $1.5 \mathrm{mM} \mathrm{MgCl} 2,250 \mu \mathrm{M}$ of dNTPs, $250 \mathrm{nM}$ of each primer, $0.25 \mathrm{U}$ of Taq polymerase (Takara, Dalian), 1xLC Green (Biochem, Salt Lake City, USA), 20 ng of genomic DNA and PCR grade water. Cycling condition was as follows: one cycle of $95^{\circ} \mathrm{C}$ for $2 \mathrm{~min}$; 45 cycles of $95^{\circ} \mathrm{C}$ for $30 \mathrm{sec}, 61^{\circ} \mathrm{C}$ for $10 \mathrm{sec}$ and $72^{\circ} \mathrm{C}$ for $30 \mathrm{sec}$. Melting curves from 72 to $95^{\circ} \mathrm{C}$ were generated by LightScanner HRI 96 (Idaho Technology Inc, Salt Lake City, USA).

DNA sequencing. To confirm HRMA results, all identified samples were sequenced (Takara, Dalian). Each sample was amplified using the same primers and condition as described in HRMA. Purified PCR products were sequenced using Big Dye Terminator v3.1 cycle sequencing kit (Applied Biosystems) according to the manufacturer's protocol. The reverse primer was used for cycle sequencing reactions. Sequencing analysis was performed on ABI Prism 3730xl DNA Analyzer (Applied Biosystems).

Statistical analysis. Association between KRAS mutation and clinicopathological features of all cases were analyzed by $\chi^{2}$ test. A $p<0.05$ was considered statistically significant.

\section{Results}

Examples of HRMA and sequencing. Examples of HRMA of gastric cancer samples carrying KRAS mutation and corresponding sequencing results were shown (Figs. 1-3). The temperature shifted melting curve and fluorescence difference curve were used to analyze the data. A sample, clearly separating from the wild-type sample in melting curves, was identified as a HRMA positive sample. All potential mutations identified by HRMA were confirmed by direct sequencing.

Mutation frequency of KRAS. In a total of 52 gastric adenocarcinomas, 5 (9.6\%) adenocarcinomas with KRAS mutation were found. All 5 mutated specimens were from male patients, but the association between KRAS mutation and patient sex was not significant $(\mathrm{P}=0.290)$. Frequencies of KRAS mutation were $14.3 \%(3 / 21)$ and $6.5 \%(2 / 31)$ in differentiated (well and moderate) and undifferentiated (poor and signet-ring cell) cancers $(\mathrm{P}=0.645) ; 25 \%(1 / 4)$ and $8.3 \%(4 / 48)$ in early and advanced wall penetration cancers $(\mathrm{P}=0.839)$; and were $13.3 \%(2 / 15)$ and $8.1 \%(3 / 37)$ in without and with lymph node metastasis cancers $(\mathrm{P}=0.952)$. The correlation between KRAS mutation and clinicopathological features is summarized in Table I.

Mutation type of KRAS. All detected KRAS mutations occurred at codon 12 and 13 of exon 2. Three of 5 mutations occurred at codon 12, including 2 G-to-A transversion mutations in the second base (replacing glycine by aspartic acid) and 1 G-to-T transversion mutations in the second base (replacing glycine by valine). Two mutations occurring at codon 13 were G-to-A transversion mutations in the second base (replacing glycine by aspartic acid). Clinicopathological features of the 5 cases with KRAS mutation are listed in Table II. All 3 codon 12 mutations were found in patients with lymph node metastasis and at advanced stage 
Table I. Patient characteristics and KRAS mutation frequency.

\begin{tabular}{|c|c|c|c|}
\hline \multirow{2}{*}{$\begin{array}{l}\text { Clinicopathological } \\
\text { features }\end{array}$} & \multirow[t]{2}{*}{ No. } & \multicolumn{2}{|c|}{ KRAS status } \\
\hline & & Mutation $(\mathrm{n}=5)$ & p-value \\
\hline Gender & & & 0.290 \\
\hline Male & 36 & 5 & \\
\hline Female & 16 & 0 & \\
\hline Age & & & 0.928 \\
\hline$\leq 65$ & 27 & 2 & \\
\hline$>65$ & 25 & 3 & \\
\hline Differentiation grade & & & 0.645 \\
\hline Well & 3 & 1 & Well and moderate vs. poor \\
\hline Moderate & 18 & 2 & and signet-ring cell cancer \\
\hline Poor & 26 & 2 & \\
\hline Signet-ring cell cancer & 5 & 0 & \\
\hline Wall penetration depth & & & 0.839 \\
\hline Early (T1) & 4 & 1 & \\
\hline Advanced (T2-T4) & 48 & 4 & \\
\hline Lymph node metastasis & & & 0.952 \\
\hline Absent & 15 & 2 & \\
\hline Present & 37 & 3 & \\
\hline Tumor stage & & & 1.000 \\
\hline $\mathrm{I}$ & 4 & 1 & Stage I and II \\
\hline II & 20 & 1 & vs. III and IV \\
\hline III & 22 & 2 & \\
\hline IV & 6 & 1 & \\
\hline
\end{tabular}

Table II. KRAS mutation type and patient characteristics.

\begin{tabular}{lcccccccc}
\hline \multicolumn{3}{c}{ KRAS mutation } & & & & & & \\
$\begin{array}{l}\text { Case } \\
\text { no. }\end{array}$ & Codon & $\begin{array}{c}\text { Nucleotide } \\
\text { change }\end{array}$ & $\begin{array}{c}\text { Amino acid } \\
\text { change }\end{array}$ & Gender & Age & $\begin{array}{c}\text { Differentiation } \\
\text { grade }\end{array}$ & $\begin{array}{c}\text { Wall } \\
\text { penetration } \\
\text { depth }\end{array}$ & $\begin{array}{c}\text { Lymph node } \\
\text { metastasis }\end{array}$ \\
\hline 1 & 13 & GGC_GAC & G_D & M & 61 & Well & Advanced & - \\
stage \\
10
\end{tabular}

(tumor stage III and IV), whereas all 2 codon 13 mutations were found in patients without lymph node metastasis and at early stage (tumor stage I and II).

\section{Discussion}

KRAS proteins regulate cell functions including survival, proliferation, differentiation and angiogenesis through complex signaling pathways (12). Activating mutations of KRAS can lead to excessively activated signaling pathways.
The mutually exclusive relationship between KRAS mutations and EGFR mutations determines the resistance of cancer with KRAS mutations to EGFR inhibitors. Therefore, KRAS mutation detection is important for discriminating patients that are resistant to EGFR inhibitors.

KRAS mutations have been extensively studied in various tumors. Dramatically different mutation frequencies were detected in pancreatic cancer $(69-90 \%)$, colorectal cancer (32-53\%), lung cancer (15-50\%) and gallbladder cancer (10$38 \%$ ) (13-19). In gastric cancer, the frequency of KRAS 



Figure 1. High-resolution melting curves and sequence traces for KRAS gene exon 2. (A) Temperature shifted melting curves of a sample with G12D mutation and a wild-type sample. (B) Fluorescence difference curves of the same samples depicted in A. (C) Sequencing analysis of the mutated sample depicted in A.
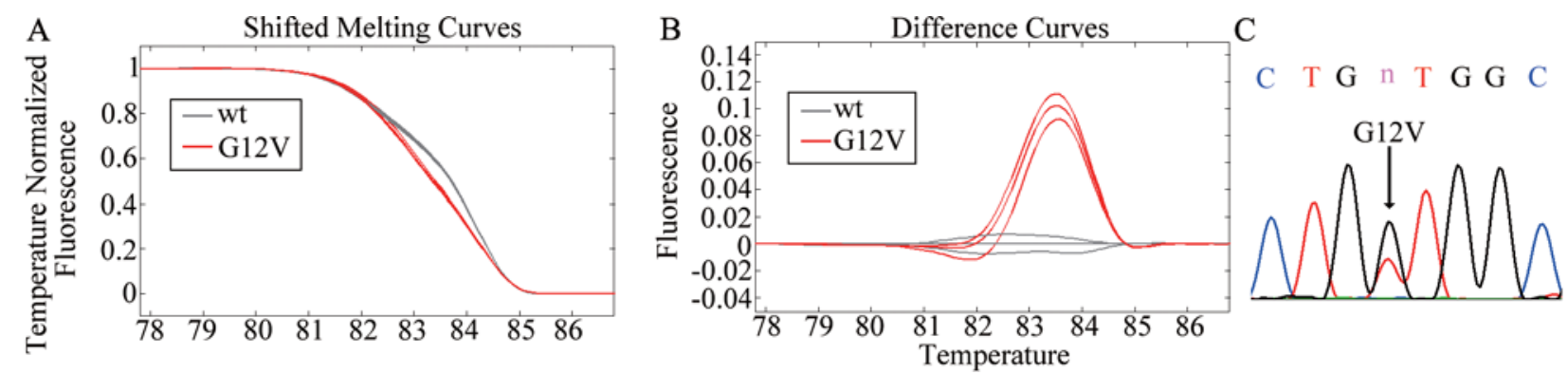

Figure 2. High-resolution melting curves and sequence traces for KRAS gene exon 2. (A) Temperature shifted melting curves of a sample with G12V mutation and a wild-type sample. (B) Fluorescence difference curves of the same samples depicted in A. (C) Sequencing analysis of the mutated sample depicted in A.
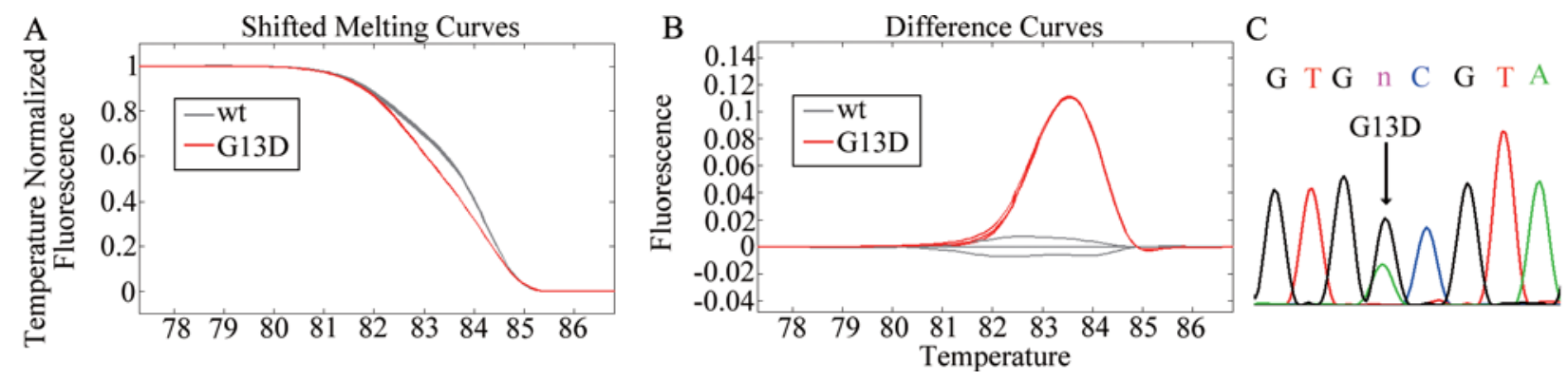

Figure 3. High-resolution melting curves and sequence traces for KRAS gene exon 2. (A) Temperature shifted melting curves of a sample with G13D mutation and a wild-type sample. (B) Fluorescence difference curves of the same samples depicted in A. (C) Sequencing analysis of the mutated sample depicted in A.

mutation being $0-21 \%(7,20)$ was relatively low, and this suggested lower expression level of KRAS gene in gastric tissue and less important role of KRAS mutation in gastric carcinogenesis. In this study, we found frequency of KRAS mutation was $9.6 \%$, which supported the evidence of low KRAS mutation frequency in gastric cancer.

Data of KRAS mutation in gastric cancer are controversial in high-frequency regions worldwide. KRAS mutation frequency of $8-21 \%$ was revealed in Italy $(7,21)$. Mutation frequencies were similar in Japan and Korea, which were $0-13 \%(22-24)$ and $2.8-9 \%$ respectively $(2,25)$. No more than $4 \%$ mutation frequency was observed in USA, Holland and Israel (26-28). No mutation was found in specimens from South Africa (20). In China, some study groups including one from Taiwan did not find KRAS mutation in gastric cancer specimens $(29,30)$, whereas $8.5 \%$ of KRAS mutation frequency was revealed by a study group from Hong Kong region (31). We found 9.6\% of KRAS mutation frequency in gastric cancers from Chinese Mainland. The result presented KRAS mutation frequency of gastric cancer in China was similar with that in other East Asian countries such as Japan and Korea. So the role of KRAS mutation in gastric carcinogenesis may be affected by geographic and racial factors. The relatively low frequency of KRAS mutation indicated KRAS mutation may only be involved in carcinogenesis of partial gastric cancer patients.

We found all 5 specimens with KRAS mutation were from male patients, suggesting KRAS mutation may play a special role in development and progression of gastric cancer in men. KRAS mutation frequency was higher in well 
differentiated than poorly differentiated samples, higher with early wall penetration than that with advanced wall penetration, higher without lymph node metastasis than with lymph node metastasis. Significant correlation between KRAS mutation and these clinicopathological features was not found, and this was consistent with previous studies $(21,32)$.

KRAS mutation usually arises at codon 12 and 13 . The spectrum of KRAS mutation types in tumors was broad. Pancreatic and colorectal cancers carried various mutation types, including G12D, G12V, G12A, G12C, G12S, G12R, G13D, G13A (13,33). However, the mutation types were relatively few in stomach, lung and gallbladder cancers, mainly G12D, G12V, G12A and G13D $(31,34,35)$. In this study, we found 3 mutation types in 5 mutations, including 2 G12D mutations, $1 \mathrm{G} 12 \mathrm{~V}$ mutation and 2 G13D mutations. The mutation types were in accordance with those found in gastric cancers from other countries. Interestingly, all codon 12 mutations were found in patients with lymph node metastasis and at advanced stage, whereas all codon 13 mutations were found in patients without lymph node metastasis and at early stage. These findings suggested codon 12 mutations of KRAS may play a role in progression of gastric cancer and codon 13 mutations may be involved in the early stage of gastric carcinogenesis. However, it is controversial on the clinicopathological features of samples with KRAS codon 12 or 13 mutation $(8,25)$. Unifying diagnosis criteria and increasing sample size may help further elucidate the role of mutations of KRAS gene codon 12 and 13 in gastric carcinogenesis.

In summary, low KRAS mutation frequency suggests KRAS mutation may only be involved in carcinogenesis of partial gastric cancer and may induce partial gastric cancer patients resistant to EGFR inhibitors. Large sample studies should be done to elucidate the KRAS mutation roles in gastric carcinogenesis and the usage of EGFR inhibitors.

\section{Acknowledgements}

We would like to thank the doctors of the Affiliated Hospitals of Dalian Medical University for the sample collection. This study was supported by DaLian Merricom gene diagnosis technology Co., Ltd.

\section{References}

1. Parkin DM, Bray F, Ferlay J and Pisani P: Estimating the world cancer burden: Globocan 2000. Int J Cancer 94: 153-156, 2001.

2. Lee SH, Lee JW, Soung YH, et al: BRAF and KRAS mutation in stomach cancer. Oncogene 22: 6942-6945, 2003.

3. Perng RP, Yang CH, Chen YM, et al: High efficacy of erlotinib in Taiwanese NSCLC patients in an expanded access program study previously treated with chemotherapy. Lung Cancer 62: 78-84, 2008.

4. Kiaris $\mathrm{H}$ and Spandidos DA: Mutations of ras genes in human tumours (Review). Int J Oncol 7: 413-421, 1995.

5. Calistri D, Rengucci C, Seymour I, et al: KRAS, p53 and BRAF gene mutations and aneuploidy in sporadic colorectal cancer progression. Cell Oncol 28: 161-166, 2006.

6. Wu CC, Hsu HY, Liu HP, et al: Reversed mutation rates of KRAS and EGFR genes in adenocarcinoma of the lung in Taiwan and their implications. Cancer 113: 3199-3208, 2008.
7. Hongyo T, Buzard GS, Palli D, et al: Mutations of the K-ras and p53 genes in gastric adenocarcinomas from a high-incidence region around Florence, Italy. Cancer Res 55: 2665-2672, 1995.

8. Hiyama T, Haruma K, Kitadai Y, et al: K-ras mutation in helicobacter pylori-associated chronic gastritis in patients with and without gastric cancer. Int J Cancer 97: 562-566, 2002.

9. Smith GD, Chadwick BE, Willmore-Payne C and Bentz JS: Detection of epidermal growth factor receptor gene mutations in cytology specimens from patients with non-small cell lung cancer utilising high-resolution melting amplicon analysis. J Clin Pathol 61: 487-493, 2008

10. Simi L, Pratesi N, Vignoli M, et al: High-resolution melting analysis for rapid detection of KRAS, BRAF, and PIK3CA gene mutations in colorectal cancer. Am J Clin Pathol 130: 247-253, 2008.

11. Krypuy M, Ahmed AA, Etemadmoghadam D, et al: High resolution melting for mutation scanning of TP53 exons 5-8. BMC Cancer 7: 168-180, 2007.

12. Adjei AA: Blocking oncogenic Ras signaling for cancer therapy. J Natl Cancer Inst 93: 1062-1074, 2001.

13. Ikeda N, Nakajima Y, Sho M, et al: The association of K-ras gene mutation and vascular endothelial growth factor gene expression in pancreatic carcinoma. Cancer 92: 488-499, 2001.

14. Wang JY, Lian ST, Chen YF, et al: Unique K-ras mutational pattern in pancreatic adenocarcinoma from Taiwanese patients. Cancer Lett 180: 153-158, 2002.

15. Kressner U, Bjørheim J, Westring S, et al: Ki-ras mutations and prognosis in colorectal cancer. Eur J Cancer 34: 518-521, 1998.

16. Moerkerk P, Arends JW, van Driel M, de Bruïne A, de Goeij A and ten Kate J: Type and number of Ki-ras point mutations relate to stage of human colorectal cancer. Cancer Res 54: 3376-3378, 1994.

17. Mascaux C, Iannino N, Martin B, et al: The role of RAS oncogene in survival of patients with lung cancer: a systematic review of the literature with meta-analysis. $\mathrm{Br} \mathrm{J}$ Cancer 92: 131-139, 2005.

18. Kohya N, Kitajima Y, Kitahara K and Miyazaki K: Mutation analysis of K-ras and beta-catenin genes related to O6-methylguanin-DNA methyltransferase and mismatch repair protein status in human gallbladder carcinoma. Int J Mol Med 11: 65-69, 2003.

19. Singh MK, Chetri K, Pandey UB, Kapoor VK, Mittal B and Choudhuri G: Mutational spectrum of K-ras oncogene among Indian patients with gallbladder cancer. J Gastroenterol Hepatol 19: 916-921, 2004.

20. Victor T, Du Toit R, Jordaan AM, Bester AJ and van Helden PD: No evidence for point mutations in codons 12,13 , and 61 of the ras gene in a high-incidence area for esophageal and gastric cancer. Cancer Res 50: 4911-4914,1990.

21. Russo A, Bazan V, Migliavacca M, et al: DNA aneuploidy and high proliferative activity but not K-ras-2 mutations as independent predictors of clinical outcome in operable gastric carcinoma: results of a 5-year Gruppo Oncologico dell'Italia Meridonale (GDIM) prospective study. Cancer 92: 294-302, 2001.

22.Endoh Y, Sakata K, Tamura G, Ohmura K, Ajioka Y, Watanabe $\mathrm{H}$ and Motoyama T: Cellular phenotypes of differentiated-type adenocarcinomas and precancerous lesions of the stomach are dependent on the genetic pathways. J Pathol 191: 257-263, 2000.

23. Tajima Y, Yamazaki K, Makino R, et al: Gastric and intestinal phenotypic marker expression in early differentiated-type tumors of the stomach: clinicopathologic significance and genetic background. Clin Cancer Res 12: 6469-6479, 2006.

24. Miki H, Ohmori M, Perantoni AO and Enomoto T: K-ras activation in gastric epithelial tumors in Japanese. Cancer Lett 58: 107-113, 1991.

25. Kim IJ, Park JH, Kang HC, et al: Mutational analysis of BRAF and K-ras in gastric cancers: absence of BRAF mutations in gastric cancers. Hum Genet 114: 118-120, 2003.

26. Lee JH, Abraham SC, Kim HS, et al: Inverse relationship between APC gene mutation in gastric adenomas and development of adenocarcinoma. Am J Pathol 161: 611-618, 2002.

27. Van Rees BP, Musler A, Caspers E, et al: K-ras mutations in gastric stump carcinomas and in carcinomas from the nonoperated stomach. Hepatogastroenterology 46: 2063-2068, 1999. 
28. Arber N, Shapira I, Ratan J, et al: Activation of c-K-ras mutations in human gastrointestinal tumors. Gastroenterology 118: 1045-1050, 2000

29. Jiang W, Kahn SM, Guillem JG, Lu SH and Weinstein IB: Rapid detection of ras oncogenes in human tumors: applications to colon, esophageal, and gastric cancer. Oncogene 4: 923-928, 1989.

30. Lin SY, Chen PH, Wang CK, et al: Mutation analysis of K-ras oncogenes in gastroenterologic cancers by the amplified created restriction sites method. Am J Clin Pathol 100: 686-689, 1993.

31. Zhao W, Chan TL, Chu KM, Chan AS, Stratton MR, Yuen ST and Leung SY: Mutations of BRAF and KRAS in gastric cancer and their association with microsatellite instability. Int $\mathrm{J}$ Cancer 108: 167-169, 2004

32. Yoo J, Park SY, Robinson RA, Kang SJ, Ahn WS and Kang CS: ras Gene mutations and expression of Ras signal transduction mediators in gastric adenocarcinomas. Arch Pathol Lab Med 126: 1096-1100, 2002.
33. Kimura K, Nagasaka T, Hoshizima N, et al: No duplicate KRAS mutation is identified on the same allele in gastric or colorectal cancer cells with multiple KRAS mutations. J Int Med Res 35: 450-457, 2007.

34. Noda N, Matsuzoe D, Konno T, Kawahara K, Yamashita Y and Shirakusa T: K-ras gene mutations in non-small cell lung cancer in Japanese. Oncol Rep 8: 889-892, 2001.

35. Rashid A, Ueki T, Gao YT, et al: K-ras mutation, p53 overexpression, and microsatellite instability in biliary tract cancers: a population-based study in China. Clin Cancer Res 8: 3156-3163, 2002 . 